$\pm N / E$

Global burnals Inc.

कै ?

\title{
Comparison of the Contamination Rate and Risk Factor Profile of Blood Culture Done in Emergency Department and MHDU/MICUs
}

\author{
By Dr. Pankaj Kumar Singh, Dr. Pranav Kumar, Dr. Mandeep Joshi, \\ Dr. Shreya Verma, Dr. Shoily Nath, Dr. Shilpi Pawar \\ \& Dr. Arpan Roy Dutta
} Abstract- Aims and objectives: To determine the risk factors of blood culture contamination done in ED and those done in the MHDU/MICU among patients admitted with medical illness.

Material and Methods: This is a two months' prospective observational study comparing blood culture contamination rate and risk factors associated with contamination between ED and MICU/MHDU. A total of 998 patients were included in the study who underwent blood culture in ED and MICU/MHDU. 570 in ED and 428 in MICU/MHDU were included after meeting exclusion and inclusion criteria.

Results: Blood culture growths were higher in ED (19\%). Most common growth was CoNS (4\%). The overall contamination rate in this study was (4.8\%) The contamination rate was lower in ED (4.4\%) when compared to MICU/MHDU (5.4\%).

Keywords: blood culture; medical intensive care unit (MICU); medical high dependency unit (MHDU); emergency departments (EDs).

GJMR-C Classification: NLMC Code: WB 105, WX 215

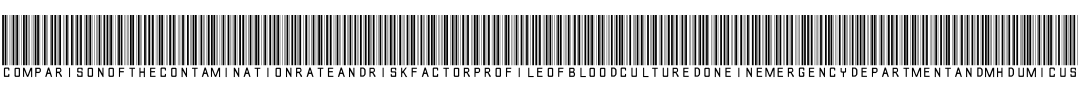

Strictly as per the compliance and regulations of:

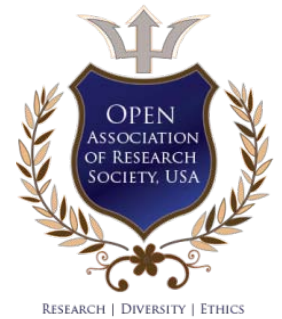

(c) 2021. Dr. Pankaj Kumar Singh, Dr. Pranav Kumar, Dr. Mandeep Joshi, Dr. Shreya Verma, Dr. Shoily Nath, Dr. Shilpi Pawar \& Dr. Arpan Roy Dutta. This is a research/review paper, distributed under the terms of the Creative Commons AttributionNoncommercial 3.0 Unported License http://creativecommons.org/licenses/by-nc/3.0/), permitting all non-commercial use, distribution, and reproduction in any medium, provided the original work is properly cited. 


\title{
Comparison of the Contamination Rate and Risk Factor Profile of Blood Culture Done in Emergency Department and MHDU/MICUs
}

\author{
Dr. Pankaj Kumar Singh ${ }^{\alpha}$, Dr. Pranav Kumar ${ }^{\sigma}$, Dr. Mandeep Joshi ${ }^{\rho}$, Dr. Shreya Verma ${ }^{\omega}$, Dr. Shoily Nath ${ }^{*}$, \\ Dr. Shilpi Pawar ${ }^{\S} \&$ Dr. Arpan Roy Dutta ${ }^{x}$
}

Abstract- Aims and objectives: To determine the risk factors of blood culture contamination done in ED and those done in the MHDU/MICU among patients admitted with medical illness.

Material and Methods: This is a two months' prospective observational study comparing blood culture contamination rate and risk factors associated with contamination between ED and MICU/MHDU. A total of 998 patients were included in the study who underwent blood culture in ED and MICU/MHDU. 570 in ED and 428 in MICU/MHDU were included after meeting exclusion and inclusion criteria.

Results: Blood culture growths were higher in ED (19\%). Most common growth was CoNS (4\%). The overall contamination rate in this study was (4.8\%) The contamination rate was lower in ED (4.4\%) when compared to MICU/MHDU (5.4\%). The Most common contaminant CoNS. The site with the least contamination rate was the dorsum of the hand (1.28\%) in ED and the most common site with contamination was femoral (22\%) in ED.

Conclusion: Emergency departments are systems particularly susceptible to a high burden of contaminated blood cultures due to high staff turnover, the need to collect cultures in critically ill patients prior to resuscitation, and the time pressure of obtaining cultures before the first dose of antibiotics. Adherence to clinical decision rules and education of EMT/Registrar is needed to improve the efficiency of blood culture taking practices.

Keywords: blood culture; medical intensive care unit (MICU); medical high dependency unit (MHDU); emergency departments (EDs).

\section{InTRODUCTION}

A s a way of identifying the organisms in the bloodstream, blood culture is a valuable method for health care practitioners. Blood cultures are an important investigation to help effective management for patients with severe infection/sepsis. A positive blood culture may indicate a conclusive diagnosis, allowing the individual organism to be targeted for therapy. However, false-positive results because of contamination can limit the utility of this important tool ${ }^{1}$. Owing to contamination, which happens when species that are not naturally present in a blood sample are grown in culture, false positives arise. For decades, contaminated cultures have been described as a

Author a: e-mail: pankajmbbsru@gmail.com problematic problem and continue to be a source of irritation for both clinical and laboratory workers. Clinicians must assess if the organism represents a clinically relevant infection associated with a high risk of morbidity and mortality or a false-positive result without any clinical effects in the face of a positive blood culture outcome. Contaminated samples increase the workload of the laboratory and can interrupt patient management or cause incorrect changes. This can prolong hospitalization of patients, increase the risk of harm, and increase health boards' costs. Current guidelines advocate a contamination rate of $2-3 \%$ is acceptable ${ }^{2}$. Emergency departments (EDs) are important locations for the diagnosis and management of bacteraemia ${ }^{3}$. Blood cultures are considered the "gold standard" for the diagnosis of bacteraemia. Emergency departments are networks that are especially vulnerable to a heavy burden of infected blood cultures due to the high turnover of workers, the need to collect cultures before resuscitation of critically ill patients, and the time pressure to acquire cultures before the first dose of antibiotics ${ }^{4}$. This study is to compare the contamination rate and risk factors of blood culture done in the emergency department and MICU/MHDU.

\section{il. Materials and Methods}

Study design: This was a prospective observational study comparing the blood culture contamination rate and risk factors in ED and MICU/MHDU.

Study setting: Christian medical college hospital, established in 1900, is a tertiary care teaching hospital situated in Vellore, Tamil Nadu. It is an important referral center in Tamil Nadu and neighboring states. The ED is one of the largest in the country and has about 74,000 admissions per year. It has a central triage system that triages all patients presenting to the ED. There are 2700 beds in the hospital of which 115 beds are allotted for ICU care. Medical Intensive care unit (MICU) and the Medical High Dependency Unit (MHDU) have 12 beds each. They receive patients directly from the ED and from Medical Wards through an open admitting system. Participants: All patients with features of bacteraemia/ fever or any infectious condition who underwent blood culture investigation in the emergency department and 
in the MICU/MHDU were recruited. Data with respect to culture methodology was collected from the ED department and MICU/MHDU.

Inclusion criteria: - Patients requiring blood culture taken in the ED. - Patients requiring blood culture in MHDU/MICU. • Patients above the age of 18. - Patients consenting to participate in the study.

Exclusion criteria: - Patients below the age of 18 . Patients NOT consenting to participate in the study. • Cultures transferred to the lab after 12 hours.

\section{Result}

Table 1: Demographic characteristics

\begin{tabular}{|c|c|c|c|c|c|}
\hline & $\begin{array}{c}\text { ED } \\
(\mathbf{n}=401)\end{array}$ & $\begin{array}{c}\text { MICU/MHDU } \\
(\mathbf{n}=379)\end{array}$ & Total (780) & P value & Cl (95\%) \\
\hline Mean age (SD) & $51.3(17.5)$ & $46.4(16.5)$ & $46.2(17.2)$ & 0.001 & $2.765-7.054$ \\
\hline Male (\%) & $340(59.6)$ & $269(62.9)$ & $478(61)$ & & \\
\hline Female (\%) & $230(40.4)$ & $159(37.1)$ & $389(39)$ & & \\
\hline
\end{tabular}

The mean age of population in the ED culture arm was 51 years and in the MICU/MHDU was 46 years. There is male predominance in both the arms. The total males accounted to $61 \%$ and the females accounted to $39 \%$.

Table 2: Demographic variables

\begin{tabular}{|c|c|c|c|}
\hline & $\begin{array}{c}\text { ED } \\
(n=570)\end{array}$ & MICU/MHDU $(n=428)$ & $\begin{array}{c}\text { Total } \\
(n=998)\end{array}$ \\
\hline \multicolumn{4}{|l|}{ Comorbidities } \\
\hline Diabetes & $209(36.7)$ & $130(30.4)$ & $339(34)$ \\
\hline Hypertension & $186(32.6)$ & 133(31.1) & $319(32)$ \\
\hline Cancer & $55(9.6)$ & $9(2.1)$ & $64(6.4)$ \\
\hline CKD & $34(6.0)$ & $63(14.7)$ & $97(9.7)$ \\
\hline CLD & $24(4.2)$ & $8(1.9)$ & $32(3.2)$ \\
\hline HIV & $9(1.6)$ & $2(0.5)$ & $11(1.1)$ \\
\hline \multicolumn{4}{|l|}{ Admission diagnosis } \\
\hline AUFI & 133(23.3) & 19(4.4) & $152(15.2)$ \\
\hline Lung infection/Pathology & $100(17.5)$ & $93(21.7)$ & 193(19.3) \\
\hline Soft tissue infection & $81(14.2)$ & $1(0.2)$ & $82(8.2)$ \\
\hline Urogenital infection & $64(11.2)$ & $11(2.6)$ & $75(7.5)$ \\
\hline Hepatobiliary pathology & $37(6.5)$ & $35(8.2)$ & $72(7.2)$ \\
\hline Haematological conditions & $23(4.0)$ & $60(14)$. & $83(8.3)$ \\
\hline Oncopathology & $24(4.2)$ & $8(1.9)$ & $32(3.2)$ \\
\hline Sepsis and septic shock & $17(3.0)$ & $60(14.0)$ & $77(7.7)$ \\
\hline Others & $91(15.9)$ & $141(32.94)$ & 182(182) \\
\hline
\end{tabular}

\section{a) Comorbidities}

The most common comorbidity in this study was diabetes comprising $36 \%$ in ED and $30 \%$ in MICU/MHDU. The second most common comorbidity was hypertension comprising $32 \%$ in $\mathrm{ED}$ and $31 \%$ in MICU/MHDU. The number of patients with CKD were more in MICU/MHDU accounting 14\%. A total of $11 \mathrm{HIV}$ cases were included in this study of which 9(1.6) in ED and 2 in MICU/MHDU.

\section{b) Admission Diagnosis}

Lung infection/Lung pathology is the most common admission diagnosis encountered in the study
Duration of study: The study was conducted for a period of 2 months from January 2019 to February 2019. Statistical analysis: Data from the Clinical Research Form was entered into epidata worksheet and the results were analysed using MS-Excel, epidata and medical. Sample size and sample size calculation: A total of 998 (570 from ED and 428 from MICU/MHDU) were recruited

comprising of 193 cases (19.3\%). However, in ED the most common admission diagnosis was AUFI comprising of $23 \%$ of total ED cases. There are no cardiac diseases in ED. Others includes neuroleptic malignant syndrome, Diphtheria infection, G6PD deficiency, post renal transplant, nephrotic syndrome, polymyositis, Liver Abscess, cardiac pathology, acute abdomen, toxicology, autoimmune diseases. 
Table 3: Blood parameters at the time of admission

\begin{tabular}{|c|c|c|c|c|}
\hline $\begin{array}{c}\text { Variable } \\
\text { (Mean/ SD) }\end{array}$ & ED $(\mathrm{n}=\mathbf{4 2 8})$ & MICU/MHDU $(\mathrm{n}=570)$ & $\mathrm{P}$ Value & $\mathrm{Cl}(\mathbf{9 5 \%})$ \\
\hline Haemoglobin & $11.82(7.29)$ & $9.89(2.74)$ & 0.001 & $1.20-2.65$ \\
\hline Total Leucocyte count & $14755(21608)$ & $13720(12911)$ & 0.380 & $-1279-3348$ \\
\hline Serum Albumin & $3.460(0.90)$ & $2.76(0.87)$ & 0.001 & $0.57-0.81$ \\
\hline
\end{tabular}

The mean value of total leucocyte counts in ED arm is higher than that of MICU/MHDU. The mean

than ED arm. Hypoalbuminemia is observed in patients with positive culture growth in MICU/MHDU. The mean serum albumin levels were lower in MICU/MHDU arm $\mathrm{Hb}$ levels were also lower in MICU/MHDU than ED arm.

Table 4: Site of poke

\begin{tabular}{|c|c|c|}
\hline Site of Poke & ED $(n=570)$ & MICU/MHDU $(n=998)$ \\
\hline Brachial & $465(81)$ & $129(30.1)$ \\
\hline Femoral & $18(3.2)$ & $1(0.2)$ \\
\hline Dorsum of hand & $78(13.7)$ & $1(0.2)$ \\
\hline Central line & $7(1.2)$ & $153(35.7)$ \\
\hline Arterial line & 0 & $144(33.6)$ \\
\hline EJV & $2(0.4)$ & 0 \\
\hline
\end{tabular}

The most common site of poke for culture in ED is brachial $81 \%$ followed by dorsum of hand $14 \%$. There was no arterial line in ED. The most common site of poke for culture in MICU/MHDU is Central line (35\%) followed by Arterial line (33\%). There was no EJV line in MICU/MHDU.

Table 5: Distribution of cases based on culture growth

\begin{tabular}{|c|c|c|c|}
\hline Culture Growth & ED $(\mathrm{n}=570)$ & MICU/MHDU $(\mathrm{n}=428)$ & Total (998) \\
\hline No growth & $462(81.1)$ & $345(80.6)$ & $807(80.9)$ \\
\hline Growth & $108(18.9)$ & $83(19.4)$ & $191(19.1)$ \\
\hline True Pathogen & $83(14.6)$ & $60(14)$ & $48(14.3)$ \\
\hline No of Contaminants & 25 & 23 & 48 \\
\hline Contamination rate & 4.4 & 5.4 & 4.8 \\
\hline
\end{tabular}

Out of 998 cases, $807(81 \%)$ showed no growth of which 462 cases are in ED and 345 cases were in MICU/MHDU. A total of 48 cases (4.8) were contaminated in the study out of which 25 cases were in $\mathrm{ED}$ and 23 in MICU/MHDU. The rate of contamination is lower in ED (4.4\%) when compared to MICU/MHDU $(5.4 \%)$. The total rate of contamination is $4.8 \%$. Out of 998 cases, 191(19\%) showed culture growth of which 108 cases are in ED and 83 were in MICU.

Table 6: Culture growth

\begin{tabular}{|c|c|c|c|}
\hline Culture growth & ED $(n=570)$ & MICU/MHDU $(n=428)$ & Total $(n=998)$ \\
\hline No growth & $462(81.1)$ & $345(80.6)$ & $807(80.9)$ \\
\hline E.coli & $20(3.5)$ & $8(1.90)$ & $28(2.80)$ \\
\hline Staph aureus & $9(1.60)$ & $4(0.90)$ & $13(1.30)$ \\
\hline Gram negative bacilli & $2(0.40)$ & $1(0.20)$ & $3(0.30)$ \\
\hline Pseudomonas & $4(0.70)$ & $3(0.70)$ & $7(0.70)$ \\
\hline Stept. Pneumoniae & $6(1.10)$ & $2(0.50)$ & $8(0.80)$ \\
\hline Proteus & $1(0.20)$ & - & $1(0.10)$ \\
\hline Candida & $1(0.20)$ & $1(0.20)$ & $2(0.20)$ \\
\hline Salmonella typhi & $1(0.20)$ & - & $1(0.10)$ \\
\hline Enterobacter species & $2(0.40)$ & - & $2(0.20)$ \\
\hline
\end{tabular}




\begin{tabular}{|c|c|c|c|}
\hline Vibrio & $1(0.20)$ & - & $1(0.10)$ \\
\hline Klebsiella & $2(0.40)$ & $12(2.80)$ & $14(1.40)$ \\
\hline Burkholderia & $1(0.20)$ & $1(0.20)$ & $2(0.20)$ \\
\hline Stenotrophomonas & $1(0.25)$ & - & $1(0.10)$ \\
\hline $\begin{array}{c}\text { Acinobacter } \\
\text { boumani }\end{array}$ & $1(0.20)$ & $1(0.20)$ & $2(0.20)$ \\
\hline Polyinfection & $1(0.20)$ & $5(1.20)$ & $6(0.60)$ \\
\hline NF- GNB & $4(0.70)$ & $14(3.3)$ & $18(1.80)$ \\
\hline Yeast & - & $1(0.20)$ & $1(0.10)$ \\
\hline Coagulase negative Staph. & $23(4.0)$ & $7(1.6)$ & $30(3.0)$ \\
\hline Viridians Streptococci & $2(0.40)$ & - & $2(0.20)$ \\
\hline Moraxella & $1(0.20)$ & - & $1(0.10)$ \\
\hline Contaminants & $25(4.4 \%)$ & $23(5.40)$ & $48(4.80)$ \\
\hline
\end{tabular}

In ED, the most common positive pathogen grown on culture was E.COLI (3.5) followed by Staph Aureus $9(1.6 \%)$ and the most common pathogen grown in MICU/MHDU arm was Nonfermenting gram negative bacilli (3.3\%) followed by Klebsiella (2.8). The total numbers of contaminants were 48 out of which 25 in ED and 23 in MICU/MHDU. $\varnothing$ During study most common contaminant was CoNS total of 26 cases out of which more were in MICU/MHDU (16 cases). $\varnothing$ Second most common contaminant was NF-GNB 1 in each department.

Table 7: Distribution of Contaminant in ED and MICU/MHDU

\begin{tabular}{|c|c|c|c|}
\hline & ED N=25 & MICU N=23 & TOTAL N=48 \\
\hline CoNS(As Contaminants) & $10(40)$ & $16(69.5)$ & $26(54.20)$ \\
\hline NF-GNB(As Contaminants) & $1(4)$ & $1(4.3)$ & $2(4.20)$ \\
\hline True Contaminant & $14(56)$ & $6(26.2)$ & $20(41.60)$ \\
\hline
\end{tabular}

Total contamination was 48. Most common True contaminants were reported (20 cases) out of contaminant was CoNS (10 cases). NF-GNB as contaminant were found 1 in each department. Total

Table 8: Contamination IN ED and MICU/MHDU

\begin{tabular}{|c|c|c|c|c|c|}
\hline \multirow{2}{*}{ Department } & \multicolumn{2}{|c|}{ Contaminants } & P Value & Odds Ratio & $95 \% \mathrm{Cl}$ \\
\hline & YES (N=48) & NO (N=950) & & & \\
\hline ED & $25(52.1 \%)$ & $545(57.4 \%)$ & 0.470 & 0.808 & $0.452-1.444$ \\
\hline MICU/MHDU & $23(47.9 \%)$ & $405(42.6 \%$ & & & \\
\hline
\end{tabular}

A total of 48 cases were contaminated in the study. IN ED 25 cases were have contamination. In MICU/MHDU 23 cases were having contamination. In

our study there was no significant difference found in contamination rate between culture done in ED and MICU/MHDU.

Table 9: Contamination based on site of poke

\begin{tabular}{|c|c|c|}
\hline Site of Poke & ED & MICU/MHDU \\
\hline Brachial & $19 / 465(4.08 \%)$ & $8 / 129(6.20 \%)$ \\
\hline Femoral & $4 / 18(22.2 \%)$ & 0 \\
\hline Dorsum of hand & $1 / 78(1.28 \%)$ & 0 \\
\hline Central line & $1 / 7(14.28 \%)$ & $9 / 153(5.88 \%)$ \\
\hline Arterial line & 0 & $6 / 144(4.16 \%)$ \\
\hline
\end{tabular}

The most common site of poke for contamination in ED was from the femoral (22.2\%) and the least common site of contamination was dorsum of hand (1.28\%). In MICU/MHDU, the most common site of contamination is from the Brachial (6.20\%) and the least common is from the arterial line (4.16\%). Arterial line blood culture sample was not done in Ed. No femoral and Dorsum of hand blood culture sample was taken in MICU/MHDU. 
Table 10: Procedure related characteristics

\begin{tabular}{|c|c|c|}
\hline Variables & $E D(n=100)$ & MICU/MHDU $(n=100)$ \\
\hline Sterile gloves & 100 & 100 \\
\hline Mask & 14 & 75 \\
\hline Wear in sterile manner & 91 & 100 \\
\hline Crowding present & 35 & 0 \\
\hline Allow to dry & 32 & 100 \\
\hline Febrile & 42 & 19 \\
\hline Scrub hand & 0 & 62 \\
\hline Plastic apron & 0 & 66 \\
\hline Head cap used & 2 & 63 \\
\hline Culture bottle top cleaned & 5 & 0 \\
\hline First Attempts & 91 & 92 \\
\hline Adequate barrier method & 14 & 64 \\
\hline $\begin{array}{c}\text { Antibiotic taken } \\
\text { prior }\end{array}$ & 9 & 47 \\
\hline Chlorhexidine & $115(20.2)$ & $426(99.5)$ \\
\hline Betadine & $455(79.8)$ & $2(0.5)$ \\
\hline Blood culture set & $443(77.7)$ & $218(50.9)$ \\
\hline Dressing set & $115(20.2)$ & $1(0.2)$ \\
\hline Others & $12(2.1)$ & $209(48.8)$ \\
\hline \multicolumn{3}{|l|}{ Volume collected } \\
\hline $5 \mathrm{cc}$ & 197(34.6) & $6(1.4)$ \\
\hline $10 c c$ & $364(63.9)$ & $420(98.1)$ \\
\hline$<5 \mathrm{cc}$ & $9(1.6)$ & $2(0.5)$ \\
\hline \multicolumn{3}{|l|}{ Culture taken by } \\
\hline EMT & $543(95.3)$ & 0 \\
\hline Registrar & $10(1.8)$ & $382(89.3)$ \\
\hline Intern & $17(3.0)$ & $46(10.3)$ \\
\hline
\end{tabular}

In our observation study it was found that there was no scrubbing of hand before the procedure in ED whereas scrub hand was found in $62 \%$ cases in MICU/MHDU. The gloves were worn in unsterile manner in 9 out of 100 cases of ED and overcrowding during venipuncture was found in 35 cases. The antiseptic used in ED was chlorhexidine (20\% cases) and betadine $(80 \%)$. The antiseptic used in MICU/MHDU was chlorhexidine in all the cases (100\%). In ED, the antiseptic was allowed to dry in 32 cases only. The set used for blood culture was blood culture set (77\%) and dressing set $(20 \%)$ in ED. The blood culture set was used in $51 \%$ cases of MICU/MHDU and other sets in $49 \%$ cases. In ED, the volume collected was $5 \mathrm{cc}$ in $35 \%$ cases and $10 \mathrm{cc}$ were collected in $64 \%$ of cases. In MICU/MHDU, 10cc volume was collected in $98 \%$ of cases and in 2\% cases < 5 cc was collected. In ED 95\% of cases, were collected by EMT, 17 by interns and 10 by registrars. Where as in MICU/MHDU most of the cultures were taken by registrars (89\%). In MICU/MHDU, 382 by registrar and 46 by interns. The blood culture was done in first attempt in $91 \%$ of cases of ED and $92 \%$ cases of MICU/MHDU.

Table 11(A): Procedure variables in ED

\begin{tabular}{|c|c|c|c|c|c|c|}
\hline \multicolumn{2}{|c|}{ Procedure variable } & \multicolumn{2}{|c|}{$\begin{array}{l}\text { ED contaminants } \\
(n=100)\end{array}$} & \multirow[t]{2}{*}{$P$ value } & \multirow[t]{2}{*}{ Odds ratio } & \multirow[t]{2}{*}{$95 \% \mathrm{Cl}$} \\
\hline & & Yes $(n=5)$ & No $(n=95)$ & & & \\
\hline \multirow[t]{4}{*}{ Mask } & No & 5 & 81 & \multirow[t]{4}{*}{1.000} & \multirow[t]{4}{*}{--} & \multirow[t]{4}{*}{--} \\
\hline & & $100 \%$ & $85.3 \%$ & & & \\
\hline & Yes & 0 & 14 & & & \\
\hline & & & $14.7 \%$ & & & \\
\hline \multirow[t]{3}{*}{ Sterile manner } & No & 0 & 9 & \multirow[t]{3}{*}{1.000} & \multirow[t]{3}{*}{--} & \multirow{3}{*}{--} \\
\hline & & & $9.5 \%$ & & & \\
\hline & Yes & 5 & 86 & & & \\
\hline
\end{tabular}




\begin{tabular}{|c|c|c|c|c|c|c|}
\hline & & $100 \%$ & $90.5 \%$ & & & \\
\hline Wear cap & No & 5 & 893 & \multirow[t]{4}{*}{1.000} & \multirow{4}{*}{--} & \multirow[t]{4}{*}{--} \\
\hline & & $100 \%$ & $97.9 \%$ & & & \\
\hline & Yes & 0 & 2 & & & \\
\hline & & & $2.1 \%$ & & & \\
\hline \multirow{4}{*}{$\begin{array}{c}\text { Adequate } \\
\text { barrier methods }\end{array}$} & No & 4 & 82 & \multirow[t]{4}{*}{0.537} & \multirow[t]{4}{*}{1.577} & \multirow{4}{*}{$\begin{array}{l}0.163- \\
15.234\end{array}$} \\
\hline & & $80.0 \%$ & $86.3 \%$ & & & \\
\hline & Yes & 1 & 13 & & & \\
\hline & & $20.0 \%$ & $13.7 \%$ & & & \\
\hline \multirow[t]{4}{*}{ Allow to dry } & No & 2 & 66 & \multirow[t]{4}{*}{0.324} & \multirow[t]{4}{*}{0.293} & \multirow{4}{*}{$\begin{array}{l}0.046- \\
1.848\end{array}$} \\
\hline & & $40 \%$ & $69.5 \%$ & & & \\
\hline & Yes & 3 & 29 & & & \\
\hline & & $60 \%$ & $30.5 \%$ & & & \\
\hline \multirow[t]{4}{*}{ Overcrowding } & Yes & 1 & 34 & \multirow[t]{4}{*}{0.655} & \multirow[t]{4}{*}{0.449} & \multirow{4}{*}{$\begin{array}{l}0.048- \\
4.176\end{array}$} \\
\hline & & $20 \%$ & $35.8 \%$ & & & \\
\hline & No & 4 & 61 & & & \\
\hline & & $80 \%$ & $64.2 \%$ & & & \\
\hline
\end{tabular}

Table 11(B): Procedure variables in ED

\begin{tabular}{|c|c|c|c|c|c|c|}
\hline \multirow{2}{*}{\multicolumn{2}{|c|}{ Procedure variable }} & \multicolumn{2}{|c|}{ ED contaminants $(n=570)$} & \multirow{2}{*}{$P$ value } & \multirow{2}{*}{$\begin{array}{l}\text { Odds } \\
\text { ratio }\end{array}$} & \multirow{2}{*}{$95 \% \mathrm{Cl}$} \\
\hline & & Yes $(n=25)$ & No $(n=545)$ & & & \\
\hline \multirow{4}{*}{ Set Used } & Others & 5 & 122 & \multirow{4}{*}{0.779} & \multirow{4}{*}{0.867} & \multirow{4}{*}{$\begin{array}{l}0.319- \\
2.357\end{array}$} \\
\hline & & $20 \%$ & $22.4 \%$ & & & \\
\hline & $\begin{array}{c}\text { Blood culture } \\
\text { set }\end{array}$ & 20 & 423 & & & \\
\hline & & $80 \%$ & $77.6 \%$ & & & \\
\hline \multirow{4}{*}{ volume } & $<=5 c c$ & 7 & 199 & \multirow{4}{*}{0.386} & \multirow{4}{*}{0.676} & \multirow{4}{*}{$\begin{array}{c}0.278- \\
1.647\end{array}$} \\
\hline & & $28 \%$ & $36.5 \%$ & & & \\
\hline & $>5 \mathrm{cc}$ & 18 & 346 & & & \\
\hline & & $72 \%$ & $63.5 \%$ & & & \\
\hline
\end{tabular}

The blood culture procedure variables done in ED were not significant with contamination ( $p$ value $>0.05$ ).

Table 12(A): Procedure variables in relation to contamination in MICU/MHDU

\begin{tabular}{|c|c|c|c|c|c|c|}
\hline \multirow{2}{*}{\multicolumn{2}{|c|}{ Procedure variable }} & \multicolumn{2}{|c|}{ MICU/MHDU $(n=100)$} & \multirow{2}{*}{$P$ value } & \multirow{2}{*}{ Odds ratio } & \multirow{2}{*}{$95 \% \mathrm{Cl}$} \\
\hline & & Yes $(n=5)$ & No $(n=95)$ & & & \\
\hline \multirow[t]{4}{*}{ Mask } & No & 1 & 24 & \multirow[t]{4}{*}{1.000} & \multirow[t]{4}{*}{1.714} & \multirow[t]{4}{*}{$0.191-15.481$} \\
\hline & & $16.7 \%$ & $25.5 \%$ & & & \\
\hline & Yes & 5 & 70 & & & \\
\hline & & $83.3 \%$ & $74.5 \%$ & & & \\
\hline \multirow[t]{4}{*}{ Scrub hand } & No & 3 & 35 & \multirow[t]{4}{*}{0.671} & \multirow[t]{4}{*}{0.593} & \multirow[t]{4}{*}{$0.113-3.101$} \\
\hline & & $50 \%$ & $37.2 \%$ & & & \\
\hline & Yes & 3 & 59 & & & \\
\hline & & $50 \%$ & $62.8 \%$ & & & \\
\hline \multirow[t]{4}{*}{ Apron use } & No & 1 & 33 & \multirow[t]{4}{*}{0.661} & \multirow[t]{4}{*}{2.705} & \multirow[t]{4}{*}{$0.303-24.131$} \\
\hline & & $16.7 \%$ & $35.1 \%$ & & & \\
\hline & Yes & 5 & 61 & & & \\
\hline & & $83.3 \%$ & $64.9 \%$ & & & \\
\hline \multirow[t]{4}{*}{ Wear cap } & No & 2 & 35 & \multirow[t]{4}{*}{1.000} & \multirow[t]{4}{*}{1.186} & \multirow[t]{4}{*}{$0.207-6.815$} \\
\hline & & $33.3 \%$ & $37.2 \%$ & & & \\
\hline & Yes & 4 & 59 & & & \\
\hline & & $66.7 \%$ & $62.8 \%$ & & & \\
\hline \multirow[t]{4}{*}{ Allow to dry } & No & 2 & 34 & \multirow[t]{4}{*}{1.000} & \multirow[t]{4}{*}{1.133} & \multirow[t]{4}{*}{$0.197-6.514$} \\
\hline & & $33.3 \%$ & $36.2 \%$ & & & \\
\hline & Yes & 4 & 60 & & & \\
\hline & & $66.7 \%$ & $63.8 \%$ & & & \\
\hline
\end{tabular}


Table 12(B): Procedure variables in relation to contamination in MICU/MHDU

\begin{tabular}{|c|c|c|c|c|c|c|}
\hline \multirow{2}{*}{\multicolumn{2}{|c|}{ Procedure variable }} & \multicolumn{2}{|c|}{ MICU/MHDU $(n=428)$} & \multirow{2}{*}{$P$ value } & \multirow{2}{*}{ Odds ratio } & \multirow{2}{*}{$95 \% \mathrm{Cl}$} \\
\hline & & Yes $(n=23)$ & No $(n=405)$ & & & \\
\hline \multirow[t]{4}{*}{ Set Used } & Others & 18 & 192 & \multirow[t]{4}{*}{0.756} & \multirow[t]{4}{*}{1.125} & \multirow[t]{4}{*}{$0.535-2.367$} \\
\hline & & $58.1 \%$ & $55.2 \%$ & & & \\
\hline & Blood culture set & 13 & 156 & & & \\
\hline & & $41.9 \%$ & $44.8 \%$ & & & \\
\hline \multirow[t]{4}{*}{ volume } & $<=5 \mathrm{cc}$ & 1 & 7 & \multirow[t]{4}{*}{0.367} & \multirow[t]{4}{*}{2.584} & \multirow[t]{4}{*}{$0.304-21.94$} \\
\hline & & $4.3 \%$ & $1.7 \%$ & & & \\
\hline & $>5 \mathrm{cc}$ & 22 & 398 & & & \\
\hline & & $95.7 \%$ & $98.3 \%$ & & & \\
\hline
\end{tabular}

The blood culture procedure variables were not significant with contamination ( $p$ value $>0.05$ ).

\section{Discussion}

This was a prospective study comparing the contamination rate and risk factor profile of blood culture done in the Emergency Department and MHDU/MICUs. The analysis contained a total of 998 cases. Out of which 570 were from ED and 428 were from MICU/MHDU. This first Indian studies looking at the rates of BCC in ED and MICU/MHDU to the best of our knowledge.

The mean age in our study in ED is 51.3 years and MICU/MDHU is 46.4 years. A similar study by Choi et al had shown a mean age of 67 years in ED and $65 y e a r s$ in general ward ${ }^{5}$. As life expectancy in India is less when compared to Singapore, the mean age in our study is less than the study done by Choi et al at Singapore ${ }^{5}$.

Our study shows a slight male predominance which is in contrast to Choi et al study where there is female predominance ${ }^{5}$. This might be because of the high female sex ratio (1:1.04) in Singapore when compared to India 6 .

The most common comorbidity in our study is diabetes accounting for $339(34 \%)$ of cases. Choi et al also showed diabetes as the most common comorbidity accounting for $163 / 400(40.8 \%)$ cases $^{5}$. There is a positive association of diabetes with culture growth in our study and study by Lee et al. ${ }^{7}$. The mean hemoglobin in this study in ED was $11.82 \mathrm{mg} / \mathrm{dl}$ which is almost equivalent to the mean hemoglobin in Choi et al study which was $12.2 \mathrm{mg} / \mathrm{dl}^{5}$. The mean total leucocyte counts in ED were higher (14.7 x109/L) when compared to Choi et al study (11.6×109/L) as most of our cases presented with high fever ${ }^{5}$.

The mean serum albumin in our study is 3.4 $\mathrm{gm} / \mathrm{dl}$ which is slightly lower than Choi et al study which was $3.6 \mathrm{gm} / \mathrm{dl}^{5}$. The total rate of contamination in our study done in ED and MICU/MHDU was $4.8 \%$. In this study, the rate of contamination is lower in ED (4.4\%) when compared to MICU/MHDU (5.4\%).

A Similar study done by Choi et al showed blood culture contamination rates were higher in ED comprising $4 \%$ when compared to general wards $(0.5 \%)^{5}$. In a study by Ramirez et al showed a blood culture contamination rate in ICU decreased from $23 \%$ to $13 \%$ by using an education-based intervention ${ }^{8}$.

Raja et al studied 11000 patients over 2 years period showed that the contamination rates were higher in ICU (31\%) when compared to ED $(20 \%)^{9}$. The Bentley et al study also found that $\mathrm{BCC}$ rates were higher in $\mathrm{ED}$ (4.74 percent), which they were able to reduce to 2 percent within a year with a simple and clear checklist and rationalizing equipment to help and not detract from this approach with a specifically specified preferred technique ${ }^{2}$. Self $\mathrm{WH}$ et al in their study was able to reduce the $\mathrm{BCC}$ rates from $4.3 \%$ to $1.7 \%$ by following a standardized, sterile process for culture collection using chlorhexidine skin antisepsis, sterile gloves, sterile drapes, and checklists ${ }^{10}$.

In our study, the growth is seen in 191/998 (19.1\%) cases. Of which growth in ED is 108 (18.9\%) and in MICU/MHDU is $83(19.4 \%)$. A higher percentage of growth in ED may be because of more number of patients in this arm. A study done by Choi et al also had near similar growth in ED $(17.5 \%)^{5}$. A similar study done in ICU by Ramirez et al showed a culture growth of $31 \%(12)$. The most common contaminant found during this study was CoNS which was similar to most of the studies ${ }^{7,9}$.

The blood culture procedure variables were not significant with contamination ( $p$-value $>0.05$ ). But according to the study by Lee et al in Taiwan, there was a strong correlation between blood culture contamination rates and the degrees of ED crowding $(P .001)^{7,11}$.

A study done by Kim et al on blood culture contamination stated that the contamination rate was $0.5 \%$ in routine sterile gloving and $0.9 \%$ in optional sterile gloving with a significant P-value. Wearing a sterile glove in an aseptic manner before venepuncture may reduce blood culture contamination ${ }^{12}$. Various studies on the BCC rate among different antiseptics showed no significant difference among the antiseptics used $^{13}$. Weinstein at el. study suggests that iodine tincture and chlorhexidine tincture are equivalent antiseptic agents for skin antisepsis in patients who require blood cultures ${ }^{14}$.

In our study CoNS are commonly isolated contaminants (26 cases) from blood cultures, however, 
they can also cause true bloodstream infections. Due to its clinical effects, this distinction is of practical significance because it can avoid the unfair use of antibiotics and the development of antimicrobial resistance. More importantly, the inability to ascertain and treat true bacteremia can prove costly to the patient, more so if the patient is critically ill or immunocompromised. A clue to the significance of CoNS-positive blood cultures is the number of positive cultures, thus more the number of positive cultures, the higher the chances of it being true bacteremia. However, this is not feasible if before beginning the patient on antimicrobial agents, only a single culture sample is collected. Quantitative blood cultures (QBCs) can aid interpretation. QBCs are cumbersome and not very feasible. On the other hand, the time-to-positivity (TTP) of blood cultures after loading in the automated systems like BacT/ Alert may be a useful surrogate test for bacterial density and interpretation of the significance of CoNS isolated from positive blood cultures ${ }^{15}$.

\section{Conclusion}

Blood culture contamination is a common clinical problem and often leads to both adverse impacts on health care and costs. We identified a low contamination rate among blood cultures collected in the adult ED at our hospital $4.4 \%$ when compared to MICU/MHDU (5.4\%). We researched the process of blood culture collection and found inconsistent methods for culture collection with recurrent breaches in aseptic technique in ED. As we know ED frequently experiences high patient volumes and crowding and that leads to making things do as soon as possible and in that way, many lapses in protocol happen and that leads to degraded performance of blood cultures, both increasing the rate of contamination and decreasing the diagnostic yield.

\section{References Références Referencias}

1. Hall KK, Lyman JA. Updated Review of Blood Culture Contamination. Clin Microbiol Rev. 2006 Oct 1; 19(4):788-802.

2. Bentley J, Thakore S, Muir L, Baird A, Lee J. A change of culture: reducing blood culture contamination rates in an Emergency Department. BMJ Qual Improv Rep. 2016; 5(1).

3. Alahmadi YM, Aldeyab MA, Mcelnay JC, Scott MG, Elhajji FWD, Magee FA, et al. Clinical and economic impact of contaminated blood cultures within the hospital setting. J Hosp Infect. 2011; 77(3): 233-6.

4. Madeo M, Jackson T, Williams C. Simple measures to reduce the rate of contamination of blood cultures in Accident and Emergency. Emerg Med J. 2005 Nov 1; 22(11):810-1.

5. Choi EC-E, Chia YH, Koh YQ, Lim CZQ, Lim JC, Ooi SBS, et al. Appropriateness of blood culture: A comparison of practices between the emergency department and general wards. Infect Dis Health. 2019 Feb; 24(1):49-55.

6. Singapore's Demographic: Sex Ratio - Males to Females | Ministry of Social and Family Development [Internet]. [Cited 2019 Jun 22]. Available from: https://www.msf.gov.sg/researchanddata/Research-and-Statistics/Pages/SingaporeDemographic-SexRatio-Males-to-Females.aspx

7. Lee C-C, Lee N-Y, Chuang M-C, Chen P-L, Chang $\mathrm{C}-\mathrm{M}, \mathrm{Ko} \mathrm{W}-\mathrm{C}$. The impact of overcrowding on the bacterial contamination of blood cultures in the ED. Am J Emerg Med. 2012 Jul; 30(6):839-45.

8. Ramirez P, Gordón M, Cortes C, Villarreal E, Perezbelles $\mathrm{C}$, Robles $\mathrm{C}$, et al. American Journal of Infection Control Blood culture contamination rate in an intensive care setting: Effectiveness of an education-based intervention. Am J Infect Control. 2015; $1-4$.

9. Raja N, O'Neill B. Blood culture contamination rates in two district general hospitals in the Southeast of England. J Patient Saf Infect Control. 2017 Jan 1; 5: 57-61.

10. Self $W H$, Speroff $T$, Grijalva CG, McNaughton CD, Ashburn J, Liu D, et al. Reducing Blood Culture Contamination in the Emergency Department: An Interrupted Time Series Quality Improvement Study. Acad Emerg Med. 2013; 20(1): 89-97.

11. Trzeciak S, Rivers EP. Emergency department overcrowding in the United States: an emerging threat to patient safety and public health. Emerg Med J EMJ. 2003 Sep; 20(5):402-5.

12. Effect of Routine Sterile Gloving on Contamination Rates in Blood Culture A Cluster Randomized Trial | Annals of Internal Medicine | American College of Physicians [Internet]. [Cited 2019 Jun 7]. Available from: https://annals.org/aim/fullarticle/746768/effectroutine-sterilegloving-contamination-rates-bloodculture-cluster-randomized

13. Liu W, Duan Y, Cui W, Li L, Wang X, Dai H, et al. Skin antiseptics in venous puncture site disinfection for preventing blood culture contamination: A Bayesian network meta-analysis of randomized controlled trials. Int J Nurs Stud. 2016 Jul; 59: 156-62.

14. Story-Roller E, Weinstein MP. Chlorhexidine versus Tincture of lodine for Reduction of Blood Culture Contamination Rates: a Prospective Randomized Crossover Study. J Clin Microbiol. 2016; 54(12): 3007-9.

15. Kassis C, Rangaraj G, Jiang Y, Hachem RY, Raad I. Differentiating culture samples representing coagulase-negative staphylococcal bacteremia from those representing contamination by use of time-to-positivity and quantitative blood culture methods. J Clin Microbiol. 2009 Oct; 47(10): 3255-60. 